\title{
Brote por Salmonella enteritidis en trabajadores de un hospital
}

\author{
Ma. Eugenia Chávez-de la Peña, M.C., M.S.P., M.E., ${ }^{(1)}$ A njarath L. Higuera-Iglesias, M.C., M.E., ${ }^{(1)}$ \\ Martha A. Huertas-Jiménez, Enf., ${ }^{(2)}$ Rosa Báez-Martínez, Enf., ${ }^{(2)}$ Josefina Morales-de León, M. en $C_{\text {. }}{ }^{(3)}$ \\ Fernando A rteaga-Cabello, Q .F.B., ${ }^{(4)}$ M. Sigfrido Rangel-Frausto, M.C., M. en C., ${ }^{(2)}$ \\ Samuel Ponce de León-Rosales, M.C., M. en C.(2)
}

\section{Chávez-de la Peña ME, Higuera-IglesiasAL, Huertas-Jiménez MA, Báez-Martínez R, Morales-de León J,Arteaga-Cabello F, Rangel-Frausto MS, Ponce de León-Rosales S. Brote por Salmonella enteritidis en trabajadores de un hospital. Salud Publica Mex 2001;43:211-216.} El texto completo en inglés de este artículo está disponible en: http://www.insp.mx/salud/index.html

\section{Resumen}

Objetivo. D escribir y determinar las causas de un brote de enfermedad gastrointestinal por Salmonella enteritidis, ocurrido en junio de 1998, entre el personal trabajador de la salud (TS) que labora en un hospital de tercer nivel de atención, en la ciudad de México. Material y métodos Se incluyó a todo aquel empleado que presentó diarrea o fiebre asociada a síntomas gastrointestinales, a partir del día 8 de junio de ese año, posterior a la ingestion de alimentos en el comedor del hospital (caso), y en aquellos asintomáti$\cos$ (controles) que ingirieron alimentos durante el mismo perio do y en el mismo lugar. Se les aplicó un cuestionario para conocer los alimentos ingeridos, se realizó hemocultivo a sujetos con fiebre mayor de $38^{\circ} \mathrm{C}$ y coprocultivo a todos, incluido el personal de la cocina. En el análisis estadístico se utilizó razón de momios (RM), intervalos de confianza al 95\% (IC 95\%), ji cuadrada y valor de $p=0.05$ para conocer la significancia estadística. Resultados D esarrollaron síntomas 155 TS, y de éstos 129 completaron la encuesta; se encuestaron además 150 TS asintomáticos. LoS síntomas más comunes fueron diarrea $(85 \%)$, dolor abdominal $(84 \%)$, cefalea $(81.4 \%)$, náusea $(78.3 \%)$ y escalofríos

\author{
Chávez-de la Peña ME, Higuera-IglesiasAL, \\ Huertas-Jiménez MA, Báez-Martínez R, \\ Morales-de León J,Arteaga-Cabello F, \\ Rangel-Frausto MS, Ponce de León-Rosales S. \\ An outbreak of Salmonella gastroenteritis \\ among hospital workers. \\ Salud Publica Mex 2001;43:211-216. \\ The English version of this paper \\ is available at: http://www.insp.mx/salud/index.html
}

\begin{abstract}
A bstract
Objective. To describe and identify the causes of an outbreak of Salmonella enteritidis gastroenteritis that took place in June 1998, among tertiary care hospital workers, in Mexico City. Material and methods. Cases were hospital workers who developed diarrhea or fever associated with gastrointestinal symptoms, after a meal at the hospital's dining room on June eight; controls were asymptomatic employees who also ate at the hospital's dining room on the same day. A food questionnaire was applied, and stool samples were obtained from all study subjects, including kitchen personnel. Blood cultures were practiced for febrile patients. 0 dds ratios with $95 \%$ confidence intervals $(95 \% \mathrm{CI})$ and the chi-squared were used for statistical analysis. Statistical significance was set at $p<0.05$. Results 0 nehundred-fifty-five workers developed symptoms, but only $129(83.2 \%)$ answered the questionnaire; 150 controls were also studied. The most common symptoms were diarrhea $(85 \%)$, abdominal pain $(84 \%)$, cephalea $(81.4 \%)$, nausea $(78.3 \%)$, and chills $(74.4 \%)$. Eight blood cultures were negative; 59 stool cultures (46\%) from cases and six (4\%) from controls, were positive for Salmonella enteritidis. Egg-covered
\end{abstract}

Este trabajo se presentó en el XXIII Congreso Anual de laAsociación Mexicana de Infectología y Microbiología Clínica, en la ciudad de San Luis Potosí, del 21 al 24 de octubre de 1998.

(1) Dirección General de Epidemiología, Secretaría de Salud, México.

(2) División de Epidemiología Hospitalaria y Control de Calidad de la Atención Médica, Instituto N acional de la N utrición Salvador Zubirán (IN N SZ), México.

(3) Departamento de Ciencia y Tecnología de los Alimentos, IN N SZ, México.

(4) Departamento de Infectología, IN N SZ, México.

Fecha de recibido: 27 de julio de 1999 - Fecha de aprobado: 19 de diciembre de 2000 Solicitud de sobretiros: Dr. Samuel Ponce de León Rosales.Vasco de Q uiroga 15, colonia Sección 16,Tlalpan, 14000 México, D.F., México. Correo electrónico: sponce@ quetzal.innsz.mx 
(74.4\%). 0 cho hemocultivos fueron negativos; 59 casos (46\%) y seis controles (4\%) tuvieron coprocultivos positivos a Salmonella enteritidis. De los alimentos ingeridos, las tortas de carne capeadas con huevo (RM 19.39, IC 95\% 9.09-41.4), la crema de mamey, así como el yogur fueron significativamente más frecuentes en casos que en controles. Los cultivos de los alimentos resultaron negativos. Conclusión. Muy probablemente este brote se debió a la ingestión de alimentos contaminados (tortas preparadas con huevo, papa y carne) con insuficiente cocción. Este brote enfatiza la necesidad de mantener un programa de evaluación de la calidad de los alimentos en hospitales. El texto completo en inglés de este artículo está disponible en:http://www.Insp.mx/ salud/index.html

Palabras clave: intoxicación alimentaria por Salmonella; personal de salud; México meat was the suspected source of infection (O R 19.39,95\% CI 9.09-41.4); some other foodstuffs like fruit dessert and yogurt, were significantly more frequent in cases than in controls. Food cultures were all negative. Conclusion.T his outbreak was probably caused by Salmonella-contaminated foodstuffs (egg-covered meat with potatoes) due to deficient cooking. This report shows the importance of foodquality programs for hospital meals. The English version of this paper is available at:http://www.insp.mx/salud/index.html

Key-words: Salmonella food poisoning; health personnel; Mexico as epidemias de infección intestinal por SalmoneL lla spp son uno de los problemas más comunes en todo el mundo, principalmente en instituciones de países en desarrollo. ${ }^{1,2}$

Se estima que cada año ocurre en los Estados Unidos de América (EUA) un promedio de 800000 a cuatro millones de infecciones por Salmonella, de las cuales alrededor de 500 son fatales. Aproximadamente 40000 de éstas se confirman con laboratorio y son serotipificadas por los laboratorios estatales de salud pública, los cuales a su vez informan al Centro de Prevención y Control de Enfermedades de Atlanta, Georgia (CDC, por sus siglas en inglés). ${ }^{3}$

En México este tipo de infecciones se han notificado de manera ocasional, aunque sospechamos que la frecuencia puede ser mayor, debido al escaso control que se tiene en la manufactura y distribución de alimentos. ${ }^{4} Y$ es aún menos frecuente que se dé a conocer un brote por Salmonella entre los empleados de un hospital.

A pesar de la experiencia que se tuvo con un brote similar en 1994 por Salmonella enteritidis en el mismo hospital, existen factores determinantes en la producción de enfermedades transmitidas por alimentos (ETA); dichos factores pueden estar presentes y no ser detectados por la vigilancia poco estricta en áreas de preparación o almacenamiento de alimentos.

La epidemiología de la salmonellosis nosocomial tiene algunas características particulares. ${ }^{5}$ La edad de aparición en los pacientes parece ser un factor determinante en la susceptibilidad de infectarse por Salmonella. Los brotes provocados con grandes inóculos involucran tasas de ataque elevadas y periodos cortos de incubación. ${ }^{6}$
La mayoría de los brotes gastrointestinales por Salmonella se han asociado con algunos productos avícolas como son el huevo y el pollo y, con menos frecuencia, con alimentos contaminados por manipulación. ${ }^{3-7}$

Actualmente la tasa de huevo contaminado en EUA con $S$. enteritidis se ha estimado entre 4/1 000 a 1/14 000. La administración de alimentos y drogas (FDA, por sus siglas en inglés) estima que en EUA la probabilidad de que una gallina ponedora infectada contamine al huevo es muy baja, posiblemente de uno entre $200 .^{8}$ En México no se cuenta con un dato preciso que señale la tasa de huevo contaminado.

El objetivo de este informe es describir un brote por S. enteritidis ocurrido entre el personal que labora en un hospital de tercer nivel de atención, que comió en el comedor del hospital, el 10 de junio de 1998, así como subrayar la importancia de mantener la vigilancia activa en relación con enfermedades transmitidas por alimentos en un hospital.

\section{Material y métodos}

El hospital tiene 2321 empleados, de los cuales 1290 $(55.6 \%)$, promedio por día, acuden al comedor. Se estudió a todo el personal que presentó síntomas y aquellos asintomáticos que ingirieron alimentos durante el mismo periodo y en el mismo lugar. Se realizó un estudio de casos y controles, donde caso fue todo trabajador que, a partir del 8 y hasta el 14 de junio de 1998, presentó uno o más de los siguientes síntomas: diarrea, cefalea, vómito, fiebre, escalofríos, dolor abdominal o malestar general, desarrollados con posterioridad a la ingestión de alimentos en el comedor del hospital; y control fue todo trabajador asintomático que ingirió 
alimentos en el comedor del hospital en el mismo periodo. Se aplicó un cuestionario para buscar factores de riesgo en relación con alimentos consumidos los días 8,9 y 10 de junio de dicho año, que incluyó ficha de identificación, alimentos consumidos (desayuno, comida y cena de dos días previos y el menú del día del suceso), fecha y hora de inicio de los síntomas, complicaciones, atención médica y tratamiento, así como la realización de estudios de laboratorio. Se tomaron coprocultivos a casos y controles, y hemocultivo a sujetos con fiebre mayor de $38{ }^{\circ} \mathrm{C}$. Se realizaron coprocultivos al personal de la cocina.

Se revisó la ruta crítica de los alimentos: almacenamiento, refrigeración, área de preparación y dinámica de trabajo del personal y preparadores de alimentos, así como el estado de salud de los mismos.

Los alimentos fueron analizados en el Departamento de Ciencia y Tecnología de los Alimentos del hospital; se muestrearon de manera aleatoria 10 piezas de huevo, incluyendo en la muestra huevo en buen estado y otros que presentaban sobre el cascarón fracturas, materia fecal o sangre. Los dos lotes de huevo se mezclaron; la mitad se sometió a un lavado con agua y jabón, posteriormente se secaron con una gasa estéril y se licuaron con todo y cascarón para realizar la determinación de Salmonella. La otra mitad no se lavó y se licuó con todo y cascarón para realizar la misma determinación y de acuerdo con la metodología oficial. Además se recibieron 10 molletes y $1.5 \mathrm{~kg}$ de carne de res cruda para bistec.

El queso de los molletes y la carne se analizaron para la determinación de Salmonella y Staphylococcus aureus en alimentos.

Se utilizó la razón de momios (RM), intervalos de confianza al 95\% (IC 95\%), valor de $p$ menor de $0.05 y$ ji cuadrada para conocer la significancia estadística. La información obtenida se procesó en el programa Epi Info, versión 6.3.

\section{Resultados}

Se incluyeron 155 personas sintomáticas, de las cuales 129 respondieron a la encuesta, así como 150 asintomáticos (cuadro I).

De los 129 casos captados, se tuvieron las siguientes características: 90 mujeres $(70 \%)$ y 39 hombres (30\%), entre 15 y 65 años, con una mediana de 30, media de 32 años (DE \pm 9$)$, y personal del área administrativa e intendencia en $45 \%$. El primer caso sintomático inició el día 9 a las 17:00 horas y con coprocultivo positivo el día 10 a las 9:00 horas; el último se presentó a las 7:00 horas del día 12 de junio (figura 1); el primer caso inició con sintomatología dos horas después de haber in-

\section{Cuadro I \\ Características demográficas \\ DE CASOS Y CONTROLES. INTOXICACIÓN ALIMENTARIA. Méxıco D.F., 1998}

\begin{tabular}{llc}
$\begin{array}{l}\text { Variable } \\
\text { Género } \\
\text { Masculino }\end{array}$ & Casos (129) & Controles (150) \\
\hline Femenino & $39(30.2 \%)$ & $68(45.3 \%)$ \\
$\begin{array}{l}\text { Edad } \\
\text { Media }\end{array}$ & $90(69.8 \%)$ & $82(54.7 \%)$ \\
$\begin{array}{l}\text { Coprocultivo } \\
\text { Positivo }\end{array}$ & $32( \pm 9)$ & $34( \pm 10)$ \\
& $59(46 \%)$ & $6(4 \%)$
\end{tabular}

La probabilidad de ser caso sintomático y positivo a Salmolella fue 14 veces mayor que en los controles ( $p<0.001$, RM 14.2)

gerido alimentos, y la gran mayoría, en las 22 horas ( \pm 5) posteriores a la comida. En ese día se proporcionaron 1125 comidas al personal, y 165 a médicos, de las cuales 70\% fueron servidas entre las 13:00 y 15:00 horas. Los alimentos se preparan diariamente, y se sirven de dos a tres platillos diferentes por turno.

Los principales signos y síntomas presentados en los casos fueron: diarrea $112(85 \%)$, dolor abdominal $108(84 \%)$, cefalea $105(81.4 \%)$, náusea $101(78.3 \%)$ y escalofríos $96(74.4 \%)$. El promedio de evacuaciones diarreicas fue de seis en 24 horas; de éstas, $47 \%$ fueron líquidas, y el resto, pastosas. Requirieron de observación de más de 24 horas 18 pacientes, de los cuales tres presentaron importante desequilibrio hidroelectrolítico. El resto del personal sintomático recibió atención médica, así como la administración de antibióticos, hidratación oral o parenteral y tratamiento sintomático.

De los casos y controles que ingirieron algún alimento en el comedor del hospital el día del brote (10 de junio) durante el desayuno, se observó una relación significativa con el yogur con fruta: 12/24 casos vs. $5 / 51$ controles, con una RM de 3.68 y un IC 95\% de 1.18 - 11.51; de los alimentos ingeridos en la comida, los únicos con riesgo significativo fueron las tortas de carne: $115 / 124$ casos vs. 56/141 controles, con una RM de 19.39 y un IC $95 \%$ de 9.09-41.4, y la crema de mamey: 68/124 casos vs. 58/141 controles, con una RM de 1.74 y un IC $95 \%$ de 1.07-2.83 (cuadro II).

Se analizaron 122 muestras de coprocultivos, y resultaron positivas para Salmonella spp 71 (58\%), todas con resistencia exclusiva a nitrofurantoína. De los ocho hemocultivos tomados, en ninguno se observó crecimiento. 




Casos

Fuente: Encuesta de brote hospitalario

Figura 1. Curva epidémica por fecha y hora de síntomas. Intoxicación alimentaria. México, D.F., 1998

De los casos captados mediante la encuesta, 59 $(46 \%)$ fueron positivos a Salmonella, y 34 (26\%), negativos; no se obtuvo muestra de $36(28 \%)$. De los controles captados se tuvieron seis (4\%) positivos, 49 (33\%) negativos y $95(63.3 \%)$ no tuvieron muestra (RM 14.2, IC 95\% 5.5-36.5).

De los alimentos analizados por el laboratorio, el huevo y el queso resultaron negativos a Salmonella; sin embargo, el queso mostró crecimiento de 23000 UFC/g a Staphylococcus aureus, cuando el valor de referencia máximo es $100 \mathrm{UFC/g}$. La carne de res (bistec crudo) fue negativa a ambos gérmenes. Sin embargo, no fue posible cultivar los ingredientes de las tortas de carne, la crema de mamey y el yogur con fruta, ya que estos alimentos fueron consumidos en su totalidad el día del brote.

Se tomó coprocultivo a todo el personal de la cocina, y cinco asintomáticos resultaron positivos a Salmonella.

Cuando se revisó el área de preparación de alimentos, se observó que algunos de los perecederos ya tenían tiempo de almacenamiento e inadecuada refrigeración. El área física se encontró con higiene deficiente, mala ventilación y extracción, y el ambiente se percibía sucio en general; los preparadores de alimentos no contaban con cubrebocas ni cofia.

El personal de cocina tiene una alta rotación. Además, todo aquel que trabaja en la cocina es revisado periódicamente con estudios de laboratorio como

\section{Cuadro II}

Distribución de ALIMENTOS Y ANÁliSIS DE RIESGOS. Intoxicación ALIMEnTARIA. México D.F., 1998

\begin{tabular}{lrrrrrr} 
& $\begin{array}{c}\text { Expuestos } \\
\text { Enft }\end{array}$ & Enf- & No expuestos & & \\
& & & & & & \\
$\begin{array}{l}\text { Cenft } 9 \text { de junio } \\
\text { A sado }\end{array}$ & 3 & 8 & 8 & 19 & 0.8 & $0.17-3.83$ \\
\hline Molletes & 5 & 16 & 6 & 11 & 0.57 & $0.14-2.35$ \\
\hline Café & 5 & 15 & 6 & 12 & 0.67 & $0.16-2.73$
\end{tabular}

Desayuno 10 de junio

\begin{tabular}{lrrrrrr} 
Yogur con fruta & 12 & 5 & 30 & 46 & 3.68 & $1.18-11.51$ \\
\hline Jugo & 14 & 10 & 28 & 41 & 2.05 & $0.80-5.26$ \\
\hline Huevo & 19 & 27 & 23 & 24 & 0.73 & $0.32-1.67$ \\
\hline Pan dulce & 12 & 13 & 30 & 38 & 1.17 & $0.47-2.93$ \\
\hline Café & 19 & 24 & 23 & 27 & 0.93 & $0.41-2.11$
\end{tabular}

Comida 10 de junio

\begin{tabular}{lrrrrrl} 
Consomé & 25 & 26 & 99 & 115 & 1.12 & $0.61-2.06$ \\
\hline Sopa de fideo & 73 & 70 & 51 & 71 & 1.45 & $0.89-2.36$ \\
\hline Ensalada mixta & 40 & 51 & 84 & 90 & 0.84 & $0.50-1.4$ \\
\hline Tortas de carne & 115 & 56 & 9 & 85 & 19.39 & $9.09-41.4$ \\
\hline Carne de res & 11 & 61 & 113 & 80 & 0.13 & $0.06-0.26$ \\
\hline Crema de mamey & 68 & 58 & 56 & 83 & 1.74 & $1.07-2.83$ \\
\hline Frijoles & 35 & 30 & 89 & 111 & 1.46 & $0.83-2.55$ \\
\hline Bolillo & 30 & 24 & 94 & 117 & 1.56 & $0.85-2.84$ \\
\hline Tortillas & 18 & 36 & 106 & 105 & 0.5 & $0.26-0.93$ \\
\hline A gua sabor & 120 & 129 & 4 & 12 & 2.79 & $0.88-8.89$
\end{tabular}

Fuente: encuesta de brote hospitalario 
son: cultivos de heces, uñas y faringe. Cuatro meses previos al brote se contaba con estudios negativos de este personal.

Durante cinco meses, posteriores al brote $(10 \mathrm{de}$ junio de 1998), se dio seguimiento a toda persona positiva a Salmonella para detectar alguna sintomatología relacionada con algún proceso reactivo. De los 65 casos que se siguieron, ninguno tuvo artritis reactiva; sólo $14(21.5 \%)$ presentaron síntomas gastrointestinales inespecíficos (distensión, pesadez y diarrea ocasional). Se calculó que el costo generado para la atención médica de estos pacientes fue de aproximadamente 32970.66 pesos exclusivamente por medicamentos.

\section{Discusión}

Salmonella es la causa más común de brotes de origen alimentario. La infección se puede adquirir por la ingestión de productos avícolas (pollo y huevo, principalmente), carne, leche y vegetales, contaminados desde su origen. ${ }^{9}$ Algunos datos confirman que los brotes por S. enteritidis (44\%) son los que se encuentran más frecuentemente asociados al consumo de huevo contaminado, más que algún otro serotipo de Salmonella (15\%). ${ }^{10}$ Cada año se notifican al CDC más de 40000 infecciones por Salmonella, ${ }^{11}$ lo cual representa una fracción estimada de uno a dos millones del total de infecciones que ocurren anualmente en los EUA. ${ }^{12,13}$

Algunos brotes causados por Salmonella se han notificado en hospitales, guarderías y prisiones, ${ }^{3,4,14} \mathrm{y}$ ocurren a pesar de la vigilancia en alimentos y personal de la cocina. Los hospitales son lugares donde comúnmente se presentan estos brotes., ${ }^{3,15} \mathrm{El} 50 \%$ de infecciones nosocomiales por Salmonella, en los EUA, ocurre en guarderías de recién nacidos y pabellones pediátricos. $^{5}$

De acuerdo con la información obtenida en nuestro estudio, se puede deducir que se trata de un brote de ETA de fuente común, lo cual probablemente se debió a la ingestión de tortas de carne, principal alimento sospechoso por el valor estadístico altamente significativo que arrojó. Estas fueron elaboradas con huevo capeado, papa cocida y trozos de carne cocidos y deshebrados con una cocción insuficiente. ${ }^{16}$ Es muy probable que alguno de estos alimentos viniera ya contaminado desde su compra o se contaminara durante su preparación; desafortunadamente no se contó con la muestra de los alimentos preparados servidos en ese día ni con la materia prima con la que fueron elaborados, debido al elevado número de comensales. ${ }^{10}$ Como producto de este brote se corrigieron aspectos de supervisión y revisión de la ruta crítica en el almacenamiento, el manejo y la preparación de los alimentos. Se realizaron cambios en las instalaciones de la cocina como remodelación del área física y control estricto en la higiene del personal.

En este brote existieron condiciones adversas que pudieron haber originado la contaminación del alimento, entre ellas la compra de productos contaminados, mal almacenamiento o refrigeración y deficientes condiciones higiénicas en la preparación.

En comparación con otros bacilos gramnegativos, la Salmonella es relativamente resistente a varios factores ambientales: crece a temperaturas que oscilan entre los 8 y $45^{\circ} \mathrm{C}$, es sensible al calor y no sobrevive a temperaturas superiores a $\operatorname{los} 70^{\circ} \mathrm{C}$; es resistente a la deshidratación por años, sobre todo en las heces, el polvo y otros materiales secos como algunos alimentos para consumo humano y animal. ${ }^{17}$ El riesgo de enfermar debido a S. enteritidis por consumo de huevo se puede reducir al mejorar el intervalo de tiempo-temperatura entre la adquisición y el consumo del producto. ${ }^{8}$ Además, con un almacenamiento, una refrigeración y una cocción adecuados son destruidos cualquier tipo de gérmenes. Sin embargo, de acuerdo con el informe del análisis, el queso que se utilizó también presentó contaminación, por lo que no es posible descartar que hayan sido varios gérmenes los involucrados en el brote. En cualquier brote se puede presentar contaminación cruzada por otros alimentos contaminados o por los mismos manipuladores, ${ }^{10}$ además de la mezcla y el reciclaje de algunos de fácil descomposición. Algunos estudios han señalado que portadores asintomáticos representan un riesgo insignificante para la transmisión de Salmonella y raramente se ven involucrados como fuente de contaminación para ocasionar un brote, ${ }^{14}$ al igual que los manejadores de alimentos sintomáticos. ${ }^{7}$ No es necesario un gran inóculo $(<1$ célula $/ 100 \mathrm{~g}$ ) para desencadenar un gran brote, ${ }^{11}$ el tamaño de la dosis infectante mínima varía. En años recientes se ha demostrado que basta una concentración de 3 a 10 células por gramo de alimento para causar enfermedad. ${ }^{17}$ Sin embargo, el que portadores no se laven las manos, puede constituir un importante vehículo para la transmisión de salmonelosis nosocomial, ya que pueden contaminar alimentos, medicamentos, productos sanguíneos y equipo de hospital. ${ }^{18}$ Es importante destacar que el personal de la cocina también acudió al comedor, como el resto de los empleados, por lo que estuvo igualmente expuesto al riesgo de enfermar como los demás.

Otro aspecto importante en la actualidad es la terapia antimicrobiana; aunque generalmente $S$. enteritidis se autolimita y no requiere de la misma, algunos pacientes gravemente enfermos, inmunocomprometidos o que tienen alguna otra infección invasiva, requieren 
de una terapia antimicrobiana inmediatamente. Ampicilina, cloranfenicol y co-trimoxazole han sido los antibióticos de elección para el tratamiento contra salmonelosis, pero el incremento en el número de especies que presentan resistencia a estos antibióticos es inquietante; ${ }^{19}$ esto es común en países como España ${ }^{20}$ y en países en desarrollo donde tratan este problema de manera exitosa a base de quinolonas. ${ }^{19}$ El brote fue de una magnitud inesperada y grave sintomatología, por lo que se decidió dar tratamiento antimicrobiano que permitiera acortar la sintomatología y la diseminación de la infección al evitar portadores.

Finalmente, de acuerdo con el análisis estadístico estratificado, las tortas de carne se identificaron como el factor de riesgo más importante en la ocurrencia del brote. Un dato que llamó la atención fue el hecho de saber que una trabajadora decidió guardar su torta para llevársela a su hijo, quien enfermó después de que la comió.

Las recomendaciones proporcionadas por el CDC para prevenir infecciones por $S$. enteritidis asociadas a huevo se deben realizar de manera permanente.

Para asegurar la calidad del producto avícola, la industria debe adoptar un programa de control de calidad en el ámbito nacional. ${ }^{8,21}$

No se detectaron casos de artritis reactiva posentérica a pesar de que esto sucede en 1.6-3.9\% de los casos infectados, tanto por $S$. typhimurium como por $S$. enteritidis.22-24

\section{Referencias}

1. N avarrete S, Santos JI. Gastroenteritis. En: $N$ avarrete S, Muñoz 0 , Santos Preciado Jl, eds. Infecciones intrahospitalarias en pediatría. México, D.F.: McG raw-Hill Interamericana, 1998;137-142.

2.Temporado S, Hughes JM, Jarvis W R. N osocomial gastrointestinal infections. En:W enzel RP, ed. Prevention and control of nosocomial infections. Maryland:W illiams \& W ilkins, 1997;925-975.

3. Glynn MK, Bopp C, D ewitt W, Dabney P, Mokhtar M, Angulo FJ. Emergence of multidrug-resistant Salmonella enterica serotype typhimurium DT104 infections in the U nited States. N Engl J Med 1998;338:1333-1338. 4. Molina J, Ponce-de León S, Guerrero ML, C arvalho A, Romero C, Báez R et al. Salmonella gastroenteritis outbreak among workers from a tertiary care hospital in Mexico City. Rev Invest Clin 1997;49:349-353.

5. D uPont HL.N osocomial Salmonellosis and Shigellosis. Infect Control Hosp Epidemiol 1991;12:707-709.
6. Blaser MJ, N ewman LS. A review of human salmonellosis: I. Infective dose. Rev Infect D is 1982;4:1096-1106.

7. Blaser MJ, Rafuse EM,W ells JG, Pollard RA, Feldman RA. An outbrake of Salmonellosis involving multiple vehicles. Am J Epidemiol 1981;114: 663-670.

8. Currier RW, Trock SC. Salmonella enteritidis in eggs (letter). Infect C ontrol Hosp Epidemiol 1989;10: 343-344.

9. Khuri-Bulos N A, Khalaf MAL, Shehabi A, Shami K. Foodhandler-associated salmonella outbreak in a university hospital despite routine surveillance cultures of kitchen employees. Infect C ontrol Hosp Epidemiol 1994; 15:311-314

10. St. Louis ME, Morse DL, Potter ME, D eM elfi TM, G uzewich JJ,Tauxe RV et al. The emergence of grade A eggs as a major source of Salmonella enteritidis infections: $\mathrm{N}$ ew implications for the control of salmonellosis. JAMA 1988;259:2103-2107.

11. Hedberg CW, Korlath JA, D 'A oust JY,W hite KE, Schell W L, Miller MR et al. A multistate outbreak of Salmonella javiana and Salmonella oranienburg due to consumption of contaminated cheese.JAMA 1992;268:3203-3207. 12. Buchwald DS, Blaser MJ.A review of human Salmonellosis: II. D uration of excretion following infection with non-typhi Salmonella. Rev Infect D is 1984;6:345-356.

13. Chalker RB, Blaser MJ.A review of human Salmonellosis: III. Magnitude of Salmonella infection in the U nited States. Rev Infect D is 1988;10: 111-124.

14. Alcabes $P, O$ 'Sullivan B, N adal E, Mouzon M. An outbreak of Salmonella gastroenteritis in an urban jail. Infect Control Hosp Epidemiol 1988;9: 542-547.

15. HigueraAL, Sánchez JM, H ernández M, D o mínguez SJL,A lvarez-Sánchez P, Rodríguez-Solís E et al. Brote de intoxicación alimentaria por Salmonella en donadores de sangre del Hospital Privado de la ciudad de México (resumen). Enferm Infecc Microbiol 1998; 18 (suplemento): 546.

16. Hedberg CW, David MJ, W hite KE, MacD onald KL, 0 sterholm MT. Role of egg consumption in sporadic Salmonella enteritidis and Salmonella typhimurium infections in Minnesota.J Infect D is 1993;167:107-111.

17. Gutiérrez L. Salmonella. En: Giono-Cerezo S, Escobar-Gutiérrez A,Valdespino-Gómez JL, eds. Diagnóstico de laboratorio de infecciones gastrointestinales. México, D.F.: IN D RE-SSA, 1994;219-234.

18. Tauxe RV, Hassan LF, Findeisen KO, Sharrar RG , Blake PA. Salmonellosis in nurses: Lack of transmission to patients. J Infect $D$ is 1988;157:370-373. 19. Piddock LJV, G riggs DJ, Hall MC, Jin YF. Ciprofloxacin resistance in clinical isolates of Salmonella typhimurium obtained from two patients. Antimicrob Agents Chemother 1993;37:662-666.

20. Muñoz P,D íaz MD, Rodríguez-C réixems M,Cercenado E, PeláezT, Bouza E.Antimicrobial resistance of Salmonella isolates in a Spanish hospital.Antimicrob A gents Chemother 1993;37:1200-1202.

21. Centers for D isease Control. 0 utbreaks of Salmonella Serotype Enteritidis infection associated with consumption of raw shell eggs-United States,1994-1995. MMW R Morb Mortal W kly Rep1996;45:737-742.

22. Hannu TJ, Leirisalo-Repo M. C linical picture of reactive Salmonella arthritis. J Rheumatol 1988;15:1668-1671.

23. Briem H, Evengard B, Jonsson M. Reiter's syndrome complicating Salmonella enteritidis infection. Lancet 1978;2(8080):112.

24.Trull AK, Eastmond CJ, Panayi GS, Reid TMS. Salmonella reactive arthritis: Serum and secretory antibodies in eight patients identified after a large outbreak. Br J Rheumatol 1986;25:13-19. 